\title{
SCENARIO-BASED REQUIREMENTS ELICITATION IN A PAIN-TELETREATMENT APPLICATION
}

\author{
I. Widya, R. G. A. Bults \\ University of Twente, Centre for Telematics and Information Technology \\ Remote Monitoring \& Treatment Group, The Netherlands \\ widya@ewi.utwente.nl,bults@ewi.utwente.nl \\ M. H. A. Huis in 't Veld, M. M. R. Vollenbroek-Hutten \\ Roessingh Research and Development, The Netherlands \\ r.huisintveld@rrd.nl,m.vollenbroek@rrd.nl
}

Keywords: Requirements engineering, Requirements elicitation, Telemedicine, Trial design, Goal-oriented, Task analysis.

Abstract: $\quad$ This paper proposes a way to elicit requirements in the domain of eHealth, in particular telemedicine treatment, that is in alignment with the evidence based working practice in medicine. In collaboration with ICT developers, medical professionals co-shape the intended system, which has to support the telemedicine application. These professionals develop a scenario and provide feedback to the subsequent requirements elicitation process which is based on the developed scenario. We propose a mix of methods and techniques to elicit requirements holistically to achieve the previously mentioned alignment. The requirements elicitation applies basically a top-down scenario based approach, however complemented with additional methods to overcome the inherently incompleteness of scenarios. In the proposed approach, we for example analyze treatment tasks and their goals that are not only identified or inferred from the scenario but also from the treatment protocol defined in an associated treatment trial design. This paper only addresses the early phase of requirements engineering, later phases, which require refinements of associated use cases, are beyond the scope of this paper.

\section{INTRODUCTION}

The early stages of the development of a software based system are considered crucial to a successful design (Yu, 1997). Requirements wished by users or involved stakeholders of the system to be designed have to be elicited, analyzed, negotiated, specified and evaluated in these stages. The engineering activities on requirements for e-Health application supporting systems in general, and telemedicine treatment supporting systems in particular, are challenging research topics which deserve more attention.

Design of a telemedicine application supporting system is a complex multidisciplinary effort involving medical professionals and Information, Communication and Technology (ICT) developers. Evidence based medicine (Sackett and Rosenberg, 1995) requires the collection of medical treatment evidences, each one of which typically is acquired in stages. One of these stages includes the design of trials and the trialling of the proposed treatment protocol on humans. Trial results will then become evidence for practitioners to enable evidence based treatment of their patients in the post trial phase. Trial design (Meinert, 1986) defines the proposed treatment protocol and the setting of the trial, for example the trial inclusion and exclusion criteria, which in turn define certain characteristics of participating patients like age, gender and disabilities. These trial design components are therefore important for requirements elicitation. Accordingly, ICT developers have to hook into the trial design to improve requirements elicitation. On the other hand, medical professionals who design trials (i.e. trial designers) need ICT based systems to enable teletreatment in accordance with the proposed treatment protocol. This intertwinement requires close multidisciplinary collaboration between the experts of the two domains. 
This paper discusses how to elicit requirements for a telemedicine application supporting system in a multidisciplinary collaboration setting, in which medical professionals co-shape the system to be designed. It only addresses the early phase of requirements engineering, later phases which for example address requirements on detailed look and feel of screen windows human computer interfaces are beyond the scope of this paper. In particular, this paper describes the requirements elicitation in a work-related neck-shoulder pain teletreatment trial.

The requirements elicitation basically applies a top down development approach in which a scenario plays an essential role as a common universe of discourse to bridge the collaboration gap between the trial designers (i.e. medical professionals) and the ICT developers (see also Cysneiros (2002), Go and Carroll (2004), and Van Helvert and Fowler (2003)). Since scenarios are inherently incomplete and a holistic solution is necessary in evidence based medicine, other methods have been used to complement our scenario based requirements elicitation process. We investigate constraints imposed by identified stakeholders of the trial, who are not necessarily users of the intended system. ICT developers study parts of the trial design and also cross disciplinary literature on pain treatment. Furthermore, we analyze the objectives of the treatment tasks and activities, which are not only identified or inferred from the scenario but also from the trial design. This is in line with the way of working in medicine and if viewed from the mentioned analysis perspective we apply a goal oriented requirements elicitation approach (cf. Letier and Lamsweerde (2004), Mylopoulos et al. (1991) and $\mathrm{Yu}$ (1997).

Moreover, our approach applies a mix of methods and techniques known in the area of requirements engineering or the medical domain. The considered added value of this work is therefore more on the application and the combination of the methods and techniques in a way in accordance with the working practices in medicine. Such alignment with medical working practices is considered one of the success factor conditions for usable telemedicine systems (Wootton et al., 2006).

This paper is organized as follows. The next section describes the teletreatment application. Thereafter, we discuss the scenario based requirements elicitation approach and the elicitation results. Section 4 discusses some of the lessons learned and in the last section we present the conclusion of the work.

\section{TREATMENT APPLICATION}

This paper addresses a work-related neck-shoulder pain teletreatment application of the European eTEN project MyoTel (Myofeedback-based Teletreatment service) (MyoTel, 2009). This project investigates the feasibility of deployment of a myofeedback based teletreatment service that enables patients with neck-shoulder complaints to receive personalized remotely supervised treatment during their daily activities at their place of work. It validates the teletreatment service in four European countries.

The MyoTel service uses clinical data, in particular surface-ElectroMyoGraphy (s-EMG) that represents the trapezius muscle activity. Measures of muscle activation and relaxation patterns are fed back to the patient and also presented to a remote treating myofeedback therapist. This service provides three feedback loops to enable control of patient's muscle activation and relaxation patterns (Figure 1):

- a micro-loop provides local tactile feedback in the form of vibration from a body-worn device to indicate insufficient muscle relaxation results averaged over a moving time window,

- a meso-loop provides visual feedback on a PDA screen to indicate near real time muscle activation performance of the left and the right trapezius muscles, and

- a macro-loop provides supervised feedback from a remote therapist during planned consultation sessions.

The MyoTel telemedicine system consists of the following: 1) a Body Area Network (BAN) consisting of body-worn s-EMG sensors and vibrating actuator and a gateway PDA with wireless public network access, 2) a MyoTel m-Health portal, 3) a Terminal of a therapist, and 4) a hybrid data Communication Infrastructure consisting of public and private wireless networks, the internet and optionally an intranet.

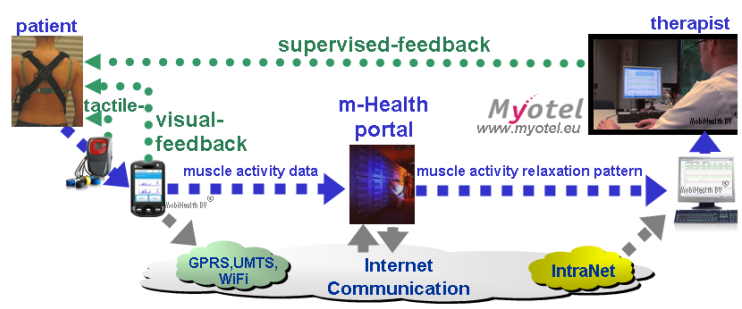

Figure 1: MyoTel neck-shoulder pain teletreatment system. 


\section{ELICITATION APPROACH}

As mentioned earlier our elicitation approach uses a mix of methods and techniques known in the literature in the area of requirements engineering or medical trial design. In this section, we describe and justify how we combine them to elicit requirements in the domain of telemedicine.

In a multidisciplinary collaboration, trial designers and ICT developers firstly develop a scenario which reflects the treatment protocol proposed in the trial design. Based on this scenario, the requirements for the intended system will be elicited. As discussed earlier, this elicitation process is complemented with a stakeholder analysis and also a task and task objective analysis. To improve the process, ICT developers study pain treatment literature and parts of the trial design.

During the elicitation process, the ICT developers conduct several e-mail exchanges and (semi) structured interviews with the trial designers. Group discussions in a participatory design setting, for example conducted during plenary project meetings, are in our case used for feedback on the scenario in development and feedback on identified (and prioritized) requirements. The elicitation, which depends on feedback of the trial designers who are myofeedback treatment professionals, uses tables, textual descriptions, and window screen mock-ups to specify the requirements. Analyzed and prioritized requirements in each iteration cycle of the early phase requirements elicitation are checked by the trial designers to validate the derived telemedicine system functionality with respect to their expectation. During these interviews and group discussions the trial designers, who are also treating therapists, therefore validate the requirements.

\subsection{Scenario}

Different definitions, scopes and objectives of scenarios are given in the literature. In this paper, we adopt the definition of Van Helvert \& Fowler (2003): "a scenario is defined as a concrete description of an activity that the users engage in when performing specific tasks, a description that is sufficiently detailed so that design implications can be inferred or reasoned about".

In accordance with Go \& Carroll (2004) but slightly adapted here, scenarios can be categorized as follows:

- Year in the life scenario: typically used in the area of strategic planning;
- Day in the life scenario: which illustrates user's daily activity, for example, in the setting of the envisioned application of the system to be designed;

- Activity in the life scenario: which describes the use of the system to be designed. These scenarios are also called use-cases, which specify user's interactions with the intended system.

Benyon \& Macaulay (2002) categorize scenarios amongst others into user-centred perspective PACT and designer-centred perspective FICS scenarios. PACT stands for People, Activity, Context and Technology, for example related to the users using a technology in their daily activity within a certain context. FICS stands for Function and events, Interactions and usability issues, Content and structure, Style and aesthetics, for example related to system use.

In the MyoTel project, we develop a day in the life scenario of a patient but combined with corresponding activities of the treating therapist (Figure 1). The purpose is to present the end-to-end interrelations of the treatment activities of the two actors that to some extent reflect the teletreatment protocol.

The development of the scenario is moreover iterative. First, the trial designers specify a day in the life scenario in terms of PACT. In the setting of a participatory design, the ICT developers provide feedback by proposing FICS extensions to the scenario. As owner of the scenario the trial designers provide an updated scenario, eventually yielding a mixed form day in the life scenario containing PACT elements combined to some extent with FICS elements. The benefits of this approach are that

- the scenario preserves its role as an embodiment of (part of) the treatment protocol and it also preserves the way of working in pain treatment as defined by the trial designers;

- ICT developers understand the implication of the scenario in respect of the required system support functionality. This is also confirmed by the trial designers who incorporate those FICS feedback in the scenario and thereby implicitly agreeing with the interpretation of the ICT developers.

In this way, the scenario acts as a common universe of discourse yielded by the discussed handshake protocol. This scenario therefore bridges the multidisciplinary collaboration gap between the trial designers and the ICT developers.

The developed scenario is shown below. 
MyoTel Scenario. Lisa is 35 years old. She is working at a large administrative company and predominantly performs computer work. She suffers from neck-shoulder pain which is, to Lisa's opinion related to the computerwork she performs because during holidays the complaints reduce. Because of this, she was allowed to have a new treatment approach; the MyoTel myofeedback treatment service. By means of the MyoTel service subjects are taught to relax their neck-shoulder muscles (socalled trapezius muscle). During her daily work, Lisa carries her Myotel BAN with her. The BAN consists of a garment, in which dry surface electrodes are incorporated, which continuously measure the muscle tension of her trapezius muscle. The garment can be worn under the clothes. The garment is connected to a processing and feedback unit which vibrates when an insufficient amount of muscle relaxation is measured. The vibration of this unit provides feedback on results (sufficient or not sufficient relaxation). Because of this vibration, Lisa knows her muscle relaxation has been insufficient for a while. In order to stop the vibrating signal, Lisa has to relax her trapezius muscle for instance by means of the relaxation exercises she has learnt from her myofeedback therapist. She is able to check her muscle relaxation patterns (parameters: Root Mean Squares (RMS) and Relative Rest Time (RRT)) for both her right and left side of her trapezius muscle live on the visual display of the PDA within the BAN. This visual information is considered to be very important as it provides Lisa more detailed information about her performance, compared to the vibration of the processing unit. The PDA also automatically sends the measured EMG or the derived muscle relaxation pattern data via a safe wireless communication infrastructure to a secured MyoTel portal which is accessible for remote consultation purposes. Lisa receives four weeks of treatment during which she wears the MyoTel BAN and notes her activities and pain intensity in a diary on the MyoTel portal. Weekly counseling sessions of approximately 30 minutes with the myofeedback therapist take place. At the start of the MyoTel treatment, Lisa and the Myotel therapist meet in vivo (face-to-face). During this so-called introduction session (in vivo), the myofeedback therapist gives instructions about the MyoTel system and explains the principles and course of remotely supervised myofeedback treatment. Lisa can read all the instructions in the manual which is provided along with the MyoTel equipment. In addition, the therapist ensures that Lisa's work station comply with the ergonomic guidelines and uses a checklist for the main work times, work tasks, working hours, workload, and work style. On the visual display of the PDA the therapist views the muscle activation patterns to check whether the garment which is worn by Lisa for the first time is properly adapted to her anatomy. Thereafter, at least three weekly remote counseling sessions take place (by telephone) in which Lisa is taught about personal work style in relation to muscle tension and beginning techniques to manage actual stressors at work and at home that may affect her musculoskeletal health. Prior to the remote counseling session (conducted by telephone), the therapist prepares the consultation. This means the therapist logs in on the web-based Myotel portal, selects Lisa from the patient list, and zooms in/out on the (historical) muscle activation patterns of the data available. The therapists seeks for differences in left and right side of the trapezius muscle, tries to find patterns in muscle relaxation over the day and/or week, identifies tasks which accompany elevated levels of muscle activation of the trapezius etcetera. In addition, screenshots of deviating or remarkable muscle activation patterns can be send to Lisa for more detailed feedback and discussion. After four weeks of treatment, Lisa visits the myofeedback therapist (in vivo) for the final counseling session and to collect the myofeedback equipment. After four weeks of treatment the pain intensity in the neck-shoulder has been reduced and Lisa is able to recognize symptoms of insufficient levels of muscle relaxation even in the absence of the service.

Refinements of the scenario towards use cases which specify detailed actor's interactions with the intended system, for example by incorporating detailed FICS elements, are beyond the scope of this early phase requirements elicitation paper.

\subsection{Complementary Elicitation Inputs}

To augment the elicitation process which is based on the developed scenario, other elicitation process inputs have been used.

\subsubsection{Cross Disciplinary Study}

ICT developers investigate literature on pain treatment and partially study trial design. The benefits of such study for these ICT developers are for example:

- understand medical vocabulary better; this is a necessity in a collaboration with trial designers who co-shape the intended system (see for similar experience also in Cysneiros (2002)); 
- get a better understanding in the clinical case, including the treatment protocol, to enable alternative solutions (cf. Cysneiros (2002)). The inclusion and exclusion criteria e.g. provide the characteristics of patients that are useful for the design of the human machine interfaces;

- $\quad$ get a better insight in the required quality of the clinical data. This helps the estimation of the required Quality of Service of data transport channels.

\subsubsection{Stakeholder Analysis}

Telemedicine typically requires holistic solutions. Demands or constraints imposed by the many different telemedicine stakeholders have to be taken into account in case that the teletreatment protocol has to be medically approved or deployed in large scale. For example, treatment protocols have to conform to medical regulations such as the regulations and guidelines of the governments, the Medical EThical Committees (METCs) or the specific association of the specialty.

In our case, the METCs in the four European countries have to approve the trial design. To anticipate to their demands, the trial design includes patient data privacy and security aspects, because regulatory stakeholders typically do not participate in the participatory design where requirements elicitation takes place. In medicine therefore, we often deal with stakeholders who are not users of the intended system. We identify and analyze the interest of these stakeholders, thereby augmenting the requirements elicitation process which is based on the scenario.

A special MyoTel stakeholder is the European eTEN Programme Commission who sponsors the trial (Section 2). This stakeholder constrains the system software adaptation, because the eTEN programme only grants minor software adaptation. Consequently, this stakeholder reduces the set of elicited requirements to those that are feasible for implementations, not only from a cost/benefit perspective but also from a software development effort perspective.

Due to the scope of the project, i.e. collecting medical evidence by trials, the identified stakeholders are the relevant ones for preparation and running the trials. Other stakeholders, for example, relevant for large scale roll out of the neckshoulder pain treatment service are not considered in this requirements elicitation process.

Stakeholders that have been identified or inferred from the scenario or the trial design are: the users stakeholder (i.e. representing both the patients and therapists), the telemedicine system provider, the communication system provider who provides the wired or wireless data transport connections, the MyoTel Centre of Excellence (CoE) who hosts the trial, the employer of the patient who provides the computing and internet access facility, the METC and to some extent the project sponsor.

\subsubsection{Role of Goals}

We adopt the scenario definition of Van Helvert \& Fowler (2003), because scenarios described in terms of tasks and activities are in accordance with working practices in medicine. We analyze and decompose the treatment tasks or activities, including their objectives (i.e. goals), to collect or infer requirements. Objectives of the treatment protocol (e.g. specified in the trial design), corresponding tasks and the associated activities (e.g. identified or inferred from the scenario) play an important role in inferring new requirements or in identifying alternative requirements. Our approach is therefore goal-oriented (Letier and Lamsweerde, 2004; Mylopoulos et al., 1991; Yu, 1997) and accordingly, we decompose goals of treatment tasks in a similar but informal way as in the previously referred papers (Figure 2).

Example. A therapist who supervises the training of a patient strictly (a task), needs amongst others to check the training discipline of the patient (a subtask, which goal is e.g. represented by SupervisedCompliance in Figure 2). Figure 2 presents a partial goal model expressed using the notation discussed in Letier and Lamsweerde (2004). The goal of managing the training compliance of a patient is represented by PatientCompliance. This goal can be achieved if amongst others SupervisedCompliance is achieved. The figure also shows that the preparation of consultation material (whose goal is represented by ConsultationMaterial) is achieved when the Activity Relaxation Patterns (ARPs) of the patient is analyzed and annotated. For this, a minimum set of measured data (mARPset) has to be available. The arrow with multiple tails in Figure 2 symbolizes an AND-refinement of the goal at the head of the arrow by the goals at the tails of the arrow (Letier and Lamsweerde, 2004), see also (Mylopoulos et al., 1991). In the goal model furthermore, the therapist may retrieve the patient's activity relaxation pattern data (PatientARPs) by operationally logging in onto the MyoTel portal (RoleBasedLogin), listing and fetching the patient's files that contain the RMS and RRT of the measured 
right and left shoulders s-EMG signals (which goals are represented by ListPatientARPs and CollectARPs, respectively; see also "tha_10" and "tha 23" in Table 1).

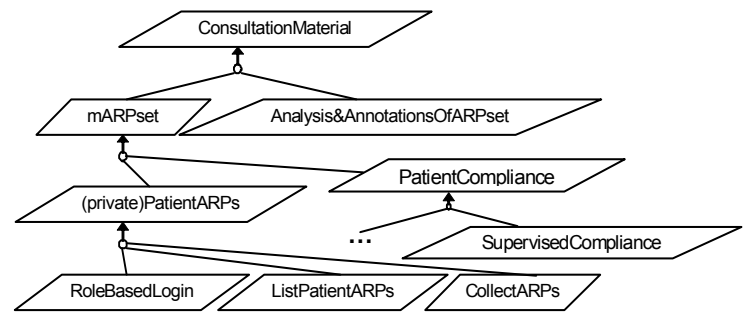

Figure 2: Consultation Material Preparation Goal Model.

\subsubsection{Requirements Elicitation Results}

A close inspection of the trial design and scenario yields the following treatment objectives:

- strictly supervised training based treatment to reduce neck-shoulder pain: from the perspective of educational science, the intended system has to facilitate remote collection and processing of training material, which are Root Mean Squares (RMS) and Relative Rest Time (RRT) of patient's left and right shoulder's s-EMGs and the three feedback mode (Section 2). Learning objective of this training is that the patient is able to recognize symptoms of insufficient muscle relaxation (see e.g. the last sentence in the scenario). A therapist supervises the training strictly, meaning that the therapist needs to check training compliance regularly and at arbitrary but convenient moments for the therapist. This moreover ensures that measured data collected in amounts and at quality needed for data analysis.

- assessment of the ICT and the diary based treatment: an objective which originates from the trial design.

Actors are users of the intended system; each of them has specific responsibility and represents an identified stakeholder. Actors that can be identified from the scenario, in particular the PACT elements, are the patients and the therapists. A close inspection of the trial design and the stakeholder analysis additionally identifies two system administrator actors. One of them represents the $\mathrm{CoE}$ trial programme owners of the participating institutes and has the responsibility to organize the trial and to administrate logistic, privacy and security aspects (as required by the METC of the CoE country). The other represents the telemedicine system provider stakeholder and has the responsibility to initialize the setting of the intended system, e.g. to register the $\mathrm{CoE}$ system administrators and to set-up the MyoTel m-Health portal (Figure 1).

Table 1: Example of Actor, Task, Activity and Priority.

\begin{tabular}{|l|l|}
\hline Actors & Tasks, Activity \& (Priority) \\
\hline myofeedback & Supervised tele-treatment task: \\
tha_10 activity: password protected \\
login to MyoTel Portal as a \\
myofeedback therapist; (need to \\
have); \\
tha_11 activity: list patient's list and \\
selects a patient; (need to have); \\
$\ldots$ \\
tha_12 activity: view selected patient \\
data with the options to zoom in/out, \\
scroll (or provide start and end \\
date/time) to view, view the \\
differences between left and right \\
muscle activation and relaxation \\
patterns. Zoom values are e.g. ... ; \\
(need to have); \\
$\ldots$ \\
tha_23 activity: check the training \\
discipline of patients by checking the \\
data on the MyoTel portal any time (cf. \\
tha_11); (need to have);
\end{tabular}

Functional needs that have to be supported by the intended system can be identified from the PACT activities of identified actors. From these functions, in turn, requirements can be derived. If the scenario contains FICS elements, the interactions induce the requirements more straightforwardly. Table 1 illustrates some of the elicited requirements for the intended system.

From the elicited requirements, ICT services that are able to support the teletreatment tasks, or associated activities, can be identified and dimensioned (cf. Van Helvert and Fowler (2003)), for example, internet data transport services to bring measured RMS or RRT data to the therapist for analysis, web services to register patients or therapists and role based login services to control data access to ensure privacy and security of data.

\section{OBSERVATION \& DISCUSSION}

Requirements elicitation in telemedicine is a challenging research topic because to some extent it has to be in alignment with the evidence based way of working in medicine. An approach which combines a holistic and a detailed in depth way of 
requirements elicitation is needed. In this work, we make use of a scenario and parts of the trial design in combination with a task and goal analysis and also a stakeholders' analysis taken from a business model perspective. This perspective has led us to the sponsoring stakeholder, which imposes additional constraints on the permitted software development effort. This stakeholder would have been missed if we had not considered the set of service provider, technology provider, organisational including regulatory and financial stakeholders. We also view treatment training as an educational science workform, which has a well defined structure in respect of objectives, roles, tasks and input/output resources in the planning, doing and evaluating phases of training. For example, patient's learning goals and needed learning material can therefore be made explicit. We consider this way of combining methods and techniques fruitful and expect that it also is typical for requirements elicitation in the domain of (diagnostic-treatment) telemedicine.

Requirements elicitation in telemedicine moreover requires an intensive collaboration between trial designers and ICT developers, for example to make topics of discussions explicit and mutually understandable. Thereby, the ICT developers need a good level of understanding of the medical vocabulary. Mutual understanding can also be improved by applying a handshake mechanism (i.e. protocol) during information exchange between trial designers and ICT developers in the iteration to strive for a common universe of discourse. In the discussed scenario based requirements elicitation process, the trial designers took the lead in the development of the scenario, which acts as the common universe of discourse in the collaboration. Thereafter, the ICT developers took the lead in the requirements elicitation process which is based on the developed and to some extent commonly understood scenario. In this way, intentions or objectives can be preserved better because the right experts manage the development.

Despite our effort to address requirements elicitation thoroughly in a multidisciplinary collaboration, we observe a limitation. Lack of cross disciplinary knowledge and homonyms of terms have caused misinterpretations. In the developed scenario, the terms "feedback on results" and feedback on performance were not recognized by the ICT developers during the requirements elicitation process as specific concepts known in the area of augmented feedback strategies. Feedback on performance (also called Knowledge of Performance $(\mathrm{KP}))$ is in some cases better than feedback on results (Knowledge of Results (KR)). Although the developed system in this perspective happens to work correctly, the ICT developers have not identified a requirement which explicitly excludes solutions that do not make sense, for example in which the ICT support for tactile KR feedback is more complex, therefore more sensitive to resource failures, than the support for visual KP feedback.

\section{CONCLUSIONS}

This paper presents a scenario based approach to elicit requirements in the domain of telemedicine that is in alignment with the evidence based working practices in medicine. Such an alignment is considered one of the success factor conditions for a usable medical system.

The scenario is developed in a multidisciplinary collaboration between medical professionals and ICT developers. Medical professionals take first the lead in the scenario development to ensure conformity to the treatment protocol and to preserve treatment objectives when the actor-activity oriented scenario is iterated and extended with actor-system interactions. Then, the ICT developers take the lead in the subsequent requirements elicitation process.

Besides scenarios, the elicitation approach makes also use of the trial design, a stakeholder's analysis and a task and goal analysis to complement the incompleteness of scenarios. The mix of methods and techniques known in the area of requirements engineering and trial design is considered the contribution of this paper.

The discussed requirements elicitation addresses the early phase of requirements engineering, later phases are considered future work for which scenarios as discussed in this paper have to be refined to use cases to detail further the actor-system interactions.

A challenging future research topic is to investigate requirements engineering for more complex treatment protocols which involve several professional caregivers who jointly manage the disease of patients, for example the treatment management of Chronic Obstructive Pulmonary Diseases (COPD).

\section{ACKNOWLEDGEMENTS}

This work has been sponsored by the EU under the eTEN programme in project MyoTel - 046230. 


\section{REFERENCES}

Benyon, D. and Macaulay, C., 2002. "Scenarios and the HCI-SE design problem", Interacting with Computers, 14, pp. 397-405;

Cysneiros, L.M., 2002. "Requirements Engineering in the Health Care Domain", in Proc. of the IEEE Joint Int. Conf. on Requirements Engineering ( $\left.R E^{\prime} 02\right)$;

Go, K. and Carroll, J.M., 2004. "The Blind Men and the Elephant: Views of Scenario-Based System Design", Interactions, pp. 45 - 53;

Letier, E. and Lamsweerde, A. van, 2004. "Reasoning about Partial Goal Satisfaction for Requirements and Design Engineering", SIGSOFT'04/FSE-12, Newport Beach, CA, Oct. 31 - Nov. 6, 2004;

Meinert, C.L., 1986. "Clinical Trial: Design, Conduct and Analysis", Oxford University Press, New York;

Mylopoulos, J., Chung, L. and Yu, E., 1991. "From Object-Oriented to Goal-Oriented Requirements Analysis", Communications of the ACM, 42, 1, Jan. 1991, pp. 31-37;

MyoTel, "MYOfeedback based TELetreatment Service", EU/eTEN programme - 046230, http://www.myotel.eu, visited Jun. 2009;

Sackett, D.L., and Rosenberg, W.M.C., 1995. "The need for evidence-based medicine", Journal of the Royal Society of Medicine, 88, pp. 620-624;

Van Helvert, J. and Fowler, C., 2003. "Scenario-based User Needs Analysis", Chimera Working Paper 200302, Colchester, University of Essex;

$\mathrm{Yu}, \mathrm{E} ., 1997$. "Towards Modelling and Reasoning Support for Early-Phase Requirements Engineering", in Proceedings of the 3rd IEEE Int. Symp. on Requirements Engineering (Washington D.C., USA, Jan. 6-8, 1997), RE'97, pp. 226-235;

Wootton, R., Craig, J., Patterson, V., 2006. "Introduction to Telemedicine", the Royal Society of Medicine Press. 